\title{
Aberrant activation of AMP-activated protein kinase remodels metabolic network in favor of cardiac glycogen storage
}

\author{
Ivan Luptak, ${ }^{1}$ Mei Shen, ${ }^{1}$ Huamei He, ${ }^{1}$ Michael F. Hirshman, ${ }^{2}$ Nicolas Musi, ${ }^{2}$ Laurie J. Goodyear, ${ }^{2}$
} Jie Yan, ${ }^{1}$ Hiroko Wakimoto, ${ }^{3}$ Hiroyuki Morita, ${ }^{3}$ Michael Arad, ${ }^{3}$ Christine E. Seidman, ${ }^{3,4}$ J.G. Seidman, ${ }^{3}$ Joanne S. Ingwall, ${ }^{1}$ James A. Balschi, ${ }^{1}$ and Rong Tian'

${ }^{1}$ NMR Laboratory for Physiological Chemistry, Division of Cardiovascular Medicine, Brigham and Women's Hospital and Harvard Medical School, Boston, Massachusetts, USA. ${ }^{2}$ Metabolism Unit, Joslin Diabetes Center and Harvard Medical School, Boston, Massachusetts, USA. ${ }^{3}$ Department of Genetics, Harvard Medical School, Boston, Massachusetts, USA. “Division of Cardiovascular Medicine, Brigham and Women's Hospital, Boston, Massachusetts, USA.

\begin{abstract}
AMP-activated protein kinase (AMPK) responds to impaired cellular energy status by stimulating substrate metabolism for ATP generation. Mutation of the $\gamma 2$ regulatory subunit of AMPK in humans renders the kinase insensitive to energy status and causes glycogen storage cardiomyopathy via unknown mechanisms. Using transgenic mice expressing one of the mutant $\gamma 2$ subunits $(\mathrm{N} 488 \mathrm{I})$ in the heart, we found that aberrant high activity of AMPK in the absence of energy deficit caused extensive remodeling of the substrate metabolism pathways to accommodate increases in both glucose uptake and fatty acid oxidation in the hearts of $\gamma 2$ mutant mice via distinct, yet synergistic mechanisms resulting in selective fuel storage as glycogen. Increased glucose entry in the $\gamma 2$ mutant mouse hearts was directed through the remodeled metabolic network toward glycogen synthesis and, at a substantially higher glycogen level, recycled through the glycogen pool to enter glycolysis. Thus, the metabolic consequences of chronic activation of AMPK in the absence of energy deficiency is distinct from those previously reported during stress conditions. These findings are of particular importance in considering AMPK as a target for the treatment of metabolic diseases.
\end{abstract}

\section{Introduction}

AMP-activated protein kinase (AMPK) is a serine/threonine kinase that acts as a cellular energy sensor and a master regulator of metabolism in a variety of cell types including cardiac myocytes (1). AMPK responds to increases in the cellular AMP/ATP ratio, an ultrasensitive indicator of impaired energy status, and triggers multiple signaling cascades to restore energy balance by stimulating ATP-generating pathways while inhibiting ATP-consuming pathways (1-3). AMPK is a heterotrimeric protein consisting of a catalytic subunit $(\alpha)$ and 2 regulatory subunits $(\beta$ and $\gamma)$. Each subunit has 2-3 isoforms; all except $\gamma 3$ are expressed in the heart. Mutations in the $\gamma 2$ subunit (encoded by the PRKAG2 gene) cause human cardiomyopathy characterized by substantial myocardial glycogen accumulation, preexcitation syndrome, and cardiac hypertrophy (4-6). These characteristics closely resemble the cardiac manifestation of glycogen storage disease (7), which prompted us to focus on the mechanisms of glycogen accumulation in the cardiomyopathy caused by mutation of PRKAG2. Interestingly, mutations in the corresponding sites on the $\gamma 3$ subunits of AMPK in skeletal muscle such as R200Q in pigs and R225Q in mice have also been shown to cause glycogen accumulation (8-10), suggesting an important role of altered glycogen metabolism in the phenotype caused by mutations of $\gamma$-AMPK.

Nonstandard abbreviations used: ACC, acetyl-CoA carboxylase; AMPK, AMPactivated protein kinase; 2-DG, 2-deoxyglucose; 2-DG-P, 2-DG-phosphate; G-6-P, glucose-6-phosphate; GS, glycogen synthase; $\gamma 2$ mutant mouse, mouse expressing the N488I mutant of $\gamma 2$-AMPK; PFK, phosphofructokinase; UDPG, UDP-linked glucose; UDPG-PPL, UDPG pyrophosphorylase; $\gamma 2$-TG mouse, transgenic mouse overexpressing WT $\gamma 2$-AMPK.

Conflict of interest: M.F. Hirshman owns stock in Johnson \& Johnson.

Citation for this article: J. Clin. Invest. 117:1432-1439 (2007). doi:10.1172/JCI30658.
In order to understand the pathogenesis of the cardiomyopathy caused by PRKAG2 mutation, transgenic mice with cardiac-specific overexpression of several mutant or WT $\gamma 2$ subunits of AMPK have been generated. Transgenic mice expressing every mutant form but WT $\gamma 2$-AMPK recapitulated the human disease phenotype, suggesting that mutation of, but not increased expression of, $\gamma 2$ subunits caused the disease phenotype (11-13). Using the mouse model expressing the N488I mutant of $\gamma 2$-AMPK in the heart (the $\gamma 2$ mutant mouse), we have previously shown that the mutation causes aberrant activation of AMPK in the absence of energy deficit and that the disease phenotype can be rescued by introducing an inactive catalytic subunit of AMPK, which acts as a dominant-negative inhibitor of AMPK $(14,15)$.

Although the disease phenotype is known to be dependent on AMPK activity, the mechanisms by which altered AMPK activity cause cardiomyopathy remain unknown. Importantly, the metabolic consequence of high AMPK activity in the energetically intact heart is not understood, despite the established role of AMPK in promoting glucose and fatty acid use during energetic stress (16-20). In the present study, we found that altered AMPK activity under normal energetic status remodeled the cardiac metabolic network to establish and turn over a substantially enlarged glycogen pool, thereby causing a unique form of glycogen storage cardiomyopathy. Thus, contrary to the role of AMPK in stimulating substrate use during stress conditions, activation of AMPK in energetically normal hearts results in fuel storage.

\section{Results}

Increased glycogen content in hearts of $\gamma 2$ mutant mice is associated with a bigher rate of glucose uptake. In the hearts of $\gamma 2$ mutant mice, there was a progressive increase in glycogen content that leveled off at 6-8 

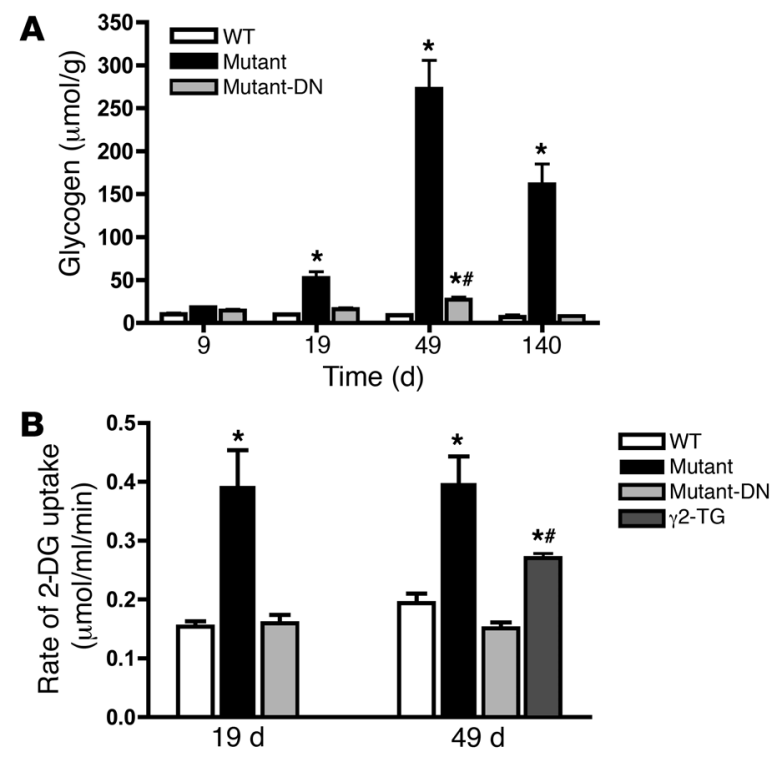

weeks of age (Figure 1A). We assessed the rate of glucose uptake in hearts of $\gamma 2$ mutant mice during the rising phase (19 days) and the plateau phase (49 days) of glycogen accumulation in isolated hearts perfused with nontracer 2-deoxyglucose (2-DG) using ${ }^{31} \mathrm{P}$ nuclear magnetic resonance (NMR) spectroscopy. The $\gamma 2$ mutant mouse hearts showed a 2-fold increase in the insulin-independent glucose uptake rate at both ages compared with nontransgenic WT controls $(P<0.05)$ and a 1.5 -fold increase compared with hearts of transgenic mice overexpressing WT $\gamma 2$-AMPK $(\gamma 2$-TG; $P<0.05$; Figure $1 \mathrm{~B})$. Crossing the $\gamma 2$ mutant mice with mice overexpressing a dominant-negative catalytic subunit of AMPK, which served to inhibit the AMPK activity in $\gamma 2$ mutant mouse hearts (14), blocked the increases in glucose uptake and glycogen content $(P<0.05$; Figure $1, \mathrm{~A}$ and $\mathrm{B})$. Thus, excessive accumulation of glycogen in the hearts of $\gamma 2$ mutant mice is associated with increased glucose uptake, and the alterations are dependent on AMPK activity and not accounted for by overexpression of the $\gamma 2$ isoform.

Increased fatty acid oxidation and decreased carbohydrate use in hearts of $\gamma 2$ mutant mice. To track the metabolic fate of increased glucose influx, we assessed the use of multiple exogenous substrates by mitochondrial oxidative metabolism in hearts of adult (7-10 weeks) $\gamma 2$ mutant and WT mice that were perfused with ${ }^{13} \mathrm{C}$-labeled substrates using ${ }^{13} \mathrm{C}$ NMR isotopomer analysis. At similar rates of myocardial oxygen consumption, the contribution of fatty acids to oxidative metabolism was $30 \%$ higher, while the contribution from carbohydrate (glucose and lactate) was more than 2 -fold lower, in hearts of $\gamma 2$ mutant mice compared with WT mice $(P<0.05$; Figure 2 , A and $\mathrm{B})$. The production of ${ }^{13} \mathrm{C}$-labeled lactate, originated from

Figure 2

Substrate use in the hearts of adult (7-10 weeks) WT and $\gamma 2$ mutant mice. Myocardial oxygen consumption $\left(\mathrm{MVO}_{2} ; \mathbf{A}\right)$ and relative contributions of each substrate to the oxidative metabolism (B) were measured in isolated mouse hearts after 45 minutes' perfusion with ${ }^{13} \mathrm{C}$-labeled substrates. (C) ${ }^{13} \mathrm{C}$-lactate output of the heart provided an estimate of glycolytic flux of ${ }^{13} \mathrm{C}$-glucose that was not coupled with oxidation. (D) Phosphorylation of ACC (p-ACC) was determined in freeze-clamped cardiac tissue. Data are mean \pm SEM $(n=5-6$ per group). ${ }^{*} P<0.05$ versus WT.

\section{Figure 1}

Glycogen content (A) and rate of glucose uptake (B) in hearts of WT, $\gamma 2$ mutant, $\gamma 2$ mutant crossed with mice overexpressing a dominantnegative catalytic subunit of AMPK (Mutant-DN), and $\gamma 2-T G$ mice at various ages. The rate of glucose uptake was assessed by a nontracer method using glucose analog 2-DG as described in Methods. Data are mean \pm SEM ( $n=4-6$ per group). ${ }^{*} P<0.05$ versus age-matched WT; ${ }^{\#} P<0.05$ versus age-matched mutant.

${ }^{13} \mathrm{C}$-labeled glucose, was markedly decreased in $\gamma 2$ mutant mouse hearts, suggesting a decreased glycolytic flux from exogenous glucose $(P<0.05$; Figure 2C). Phosphorylation of acetyl-CoA carboxylase (ACC), an endogenous substrate of AMPK, was significantly increased (Figure 2D). Because cardiac ACC (ACC $\beta$ ) catalyzes the production of malonyl-CoA, a potent inhibitor of carnitine-palmityl transferase 1 , phosphorylation and inactivation of ACC $\beta$ is consistent with the increased fatty acid oxidation observed in $\gamma 2$ mutant mouse hearts.

Increased glucose entry in hearts of $\gamma 2$ mutant mice is directed toward glycogen. Because increased glucose uptake in the hearts of $\gamma 2$ mutant mice was not associated with increased use via glycolysis or oxidation, we next tested the possibility that increased glucose entry was directed toward glycogen synthesis. We perfused the hearts of 3-week-old and 7 - to 10 -week-old mice with ${ }^{13} \mathrm{C}$-labeled glucose and measured the time-dependent accumulation of ${ }^{13} \mathrm{C}$-glycogen (Figure 3A). These 2 ages were chosen to represent the rising and peak phases of glycogen accumulation based on results shown in Figure 1A. The rate of ${ }^{13} \mathrm{C}$-glucose incorporation into the glycogen pool was $0.088 \pm 0.009$ and $0.067 \pm 0.001 \mu \mathrm{mol} / \mathrm{g} / \mathrm{min}$ in the hearts of $\gamma 2$ mutant mice at 3 weeks and 7-10 weeks, respectively. This was roughly 5 and 4 times higher, respectively, than that in hearts of WT mice at $7-10$ weeks $(0.018 \pm 0.002 \mu \mathrm{mol} / \mathrm{g} / \mathrm{min} ; P<0.05)$. We were unable to detect significant ${ }^{13} \mathrm{C}$-glucose incorporation in the hearts of 3-week-old WT mice or $\gamma 2$-TG mice; it is likely that we reached the lower limit of the instrument's sensitivity, due in part to the low rate of incorporation in these 2 groups and in part to the small heart size (50-60 mg), since NMR signals are proportionate to heart mass. Nevertheless, we were able to show that in contrast to a very low to undetectable flux in WT or $\gamma 2$-TG hearts, a robust flux of exogenous glucose toward the
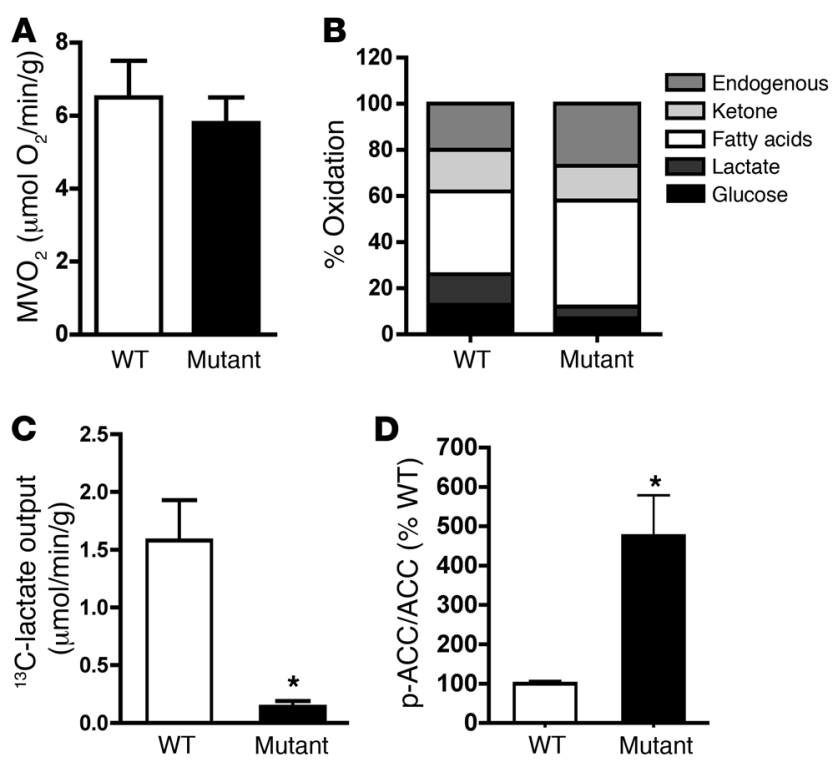

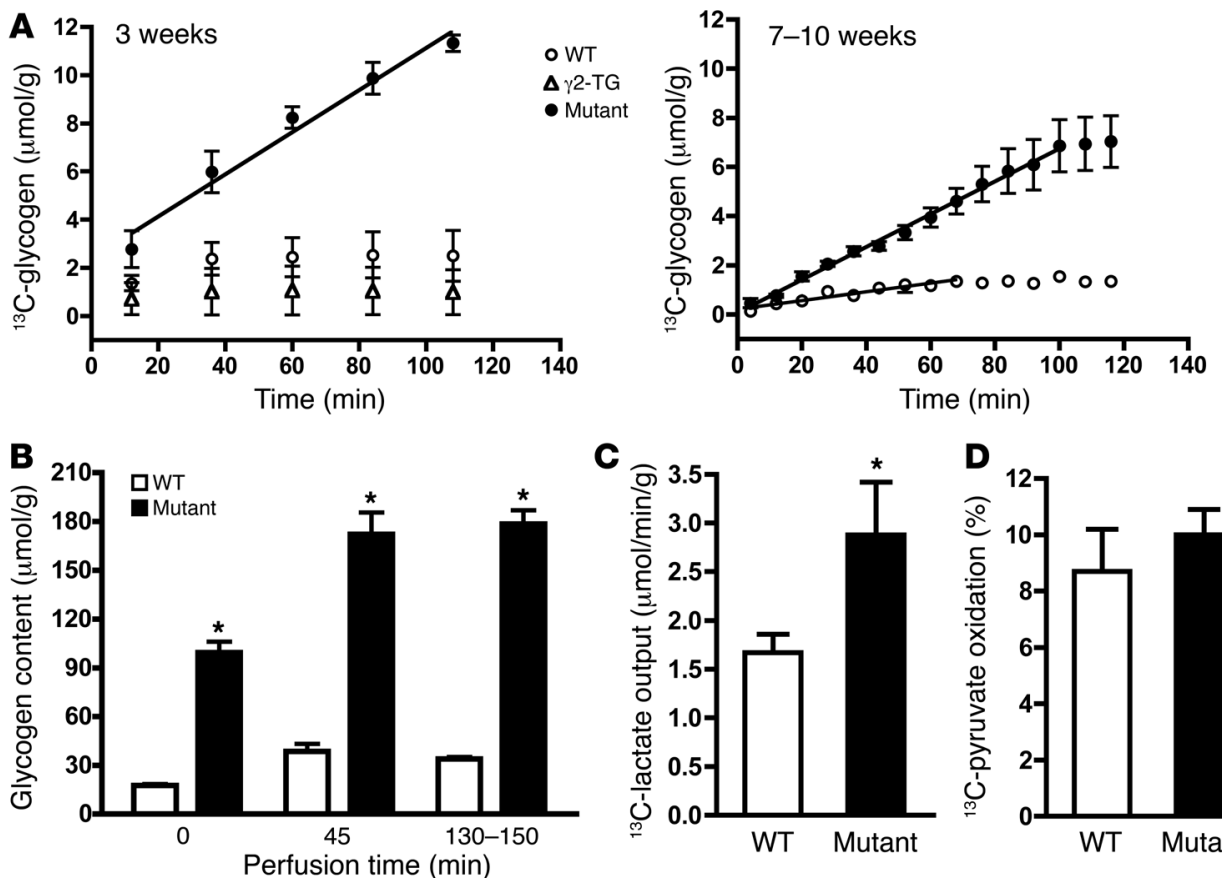

Figure 3

Glycogen metabolism in $\gamma 2$ mutant mouse hearts. (A) Time-dependent labeling of glycogen with ${ }^{13} \mathrm{C}$-glucose during 2 hours' perfusion in the hearts of WT, $\gamma 2-\mathrm{TG}$, and $\gamma 2$ mutant mice aged 3 weeks and $7-10$ weeks. Linear fit was applied to the rising phase for each group. Shown are the slopes of 3-week-old $\gamma 2$ mutant mice $\left(0.088 \pm 0.009 \mu \mathrm{mol} / \mathrm{g} / \mathrm{min} ; r^{2}, 0.995\right), 7$ - to 10 -week-old WT mice $(0.018 \pm 0.002 \mu \mathrm{mol} / \mathrm{g} /$ $\left.\min ; r^{2}, 0.916\right)$, and 7- to 10 -week-old $\gamma 2$ mutant mice $\left(0.067 \pm 0.001 \mu \mathrm{mol} / \mathrm{g} / \mathrm{min} ; r^{2}, 0.995\right)$. No significant slopes were found in 3-week-old WT hearts or $\gamma 2$-TG hearts. (B) Total glycogen content in 7- to 10-week-old WT and $\gamma 2$ mutant mouse hearts freeze-clamped at 3 time points during the glycogenlabeling process. (C and D) ${ }^{13} \mathrm{C}$-lactate output of the heart (7-10 weeks; $\mathbf{C}$ ) and the contribution of ${ }^{13} \mathrm{C}$-pyruvate, derived from glucose and glycogen, to oxidative metabolism measured at the end of 2-hour perfusion (D). Data are mean \pm SEM ( $n=5-7$ per group). ${ }^{*} P<0.05$ versus age-matched WT.

glycogen pool occurred in $\gamma 2$ mutant mouse hearts that was greater during the rising phase of glycogen accumulation $(P<0.05,3$ weeks versus $7-10$ weeks).

In hearts of 7- to 10-week-old WT mice, incorporation of ${ }^{13} \mathrm{C}$-glucose corresponded with the increase of myocardial total glycogen content: both reached a steady state with 1 hour of perfusion. In contrast, ${ }^{13} \mathrm{C}$ labeling of the glycogen pool in hearts of $\gamma 2$ mutant mice did not reach steady state until 2 hours of perfusion, despite minimal increases in total glycogen content during the second hour (Figure 3B). These results suggested that exogenous glucose was preferentially directed toward glycogen synthesis in the $\gamma 2$ mutant mouse heart and that furthermore, there was substantially higher turnover of glycogen in these hearts. Thus, we remeasured ${ }^{13} \mathrm{C}$-lactate production and glucose oxidation after 2 hours of perfusion with ${ }^{13} \mathrm{C}$-glucose, when the labeling of the glycogen pool reached steady state in the hearts of the $\gamma 2$ mutant mice. Measurements taken at this time point determined the combined contribution from exogenous glucose and glycogen to glycolysis and oxidation. Using this approach, we found that ${ }^{13} \mathrm{C}$-lactate production was $40 \%$ higher in hearts of $\gamma 2$ mutant mice compared with WT mice $(P<0.05)$, while the combined contribution of glucose and glycogen to oxidative metabolism was not different between the 2 groups (Figure 3, C and D). Comparison of results obtained after 45 minutes (Figure $2 \mathrm{C}$ ) versus 2 hours (Figure $3 \mathrm{C}$ ) of ${ }^{13} \mathrm{C}$-glucose labeling showed that approximately $90 \%$ of lactate output from the $\gamma 2$ mutant mouse hearts orig-

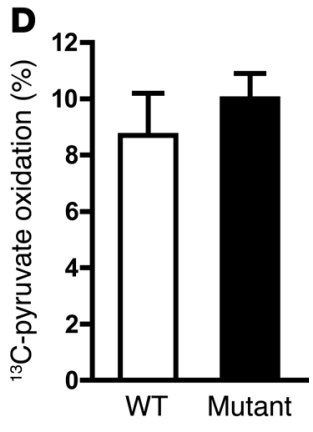

inated from glycogen. In hearts of WT mice, prolonging the duration of ${ }^{13} \mathrm{C}$ labeling did not significantly alter the results because the steady state was reached after 45 minutes of labeling. Together, these results suggested that glucose metabolism in the hearts of $\gamma 2$ mutant mice was redirected in favor of the glycogen synthesis pathway and that the glycolytic flux in the hearts of adult $\gamma 2$ mutant mice originated primarily from glycogen instead of exogenous glucose.

Mutation of cardiac $\gamma 2-A M P K$ remodels the glycogen metabolism. To further understand the mechanisms responsible for directing exogenous glucose toward the glycogen pool, we assessed the regulation of the key enzymes involved in glycogen synthesis in the hearts of WT and $\gamma 2$ mutant mice. A key step in the glycogen synthesis pathway is the addition of UDPlinked glucose (UDPG) to glycogenin. The generation of UDPG is catalyzed by UDPG pyrophosphorylase (UDPG-PPL). Northern and Western blotting analyses showed significant increases in the mRNA and protein levels of UDPG-PPL in the hearts of $\gamma 2$ mutant mice compared with WT mice (Figure $4, \mathrm{~A}$ and $\mathrm{B})$. The increase in protein expression was first observed at 1 week, which was coincident with the full activation of the $\alpha$-myosin heavy chain promoter that drove the mutant Prkag2 gene (21-23). The increase was attenuated by crossing the $\gamma 2$ mutant mice with mice overexpressing a dominant-negative catalytic subunit of AMPK (Figure 4C). The increased expression of UDPG-PPL in the hearts of $\gamma 2$ mutant mice was unlikely attributable to increased glucose entry, because the UDPG-PPL level was downregulated in transgenic hearts with elevated glucose uptake rate due to overexpression of the insulinindependent glucose transporter $1(P<0.05$; Figure 4C) (24). Furthermore, we found that UDPG-PPL activity was increased 5 -fold in $\gamma 2$ mutant mouse hearts $(P<0.05)$ and that this increase was not affected by phosphatase treatment (Figure 4D); the latter finding suggests that modification by phosphorylation was unlikely. These results collectively suggest that transcriptional mechanisms contribute to the upregulation of UDPG-PPL activity caused by mutation of $\gamma 2$-AMPK.

We next measured the activity of glycogen synthase (GS) at increasing concentrations of glucose-6-phosphate (G-6-P), the allosteric activator of the enzyme (Figure 5A). At 9 days, GS activity was not different in hearts of WT and $\gamma 2$ mutant mice over the entire range of G-6-P concentrations. At 19 and 49 days, the relationship of GS activity to G-6-P concentration was significantly altered in the $\gamma 2$ mutant mouse hearts $(P<0.05$, interaction between G-6-P concentration and genotype on GS activity; 2-way repeated-mea- 


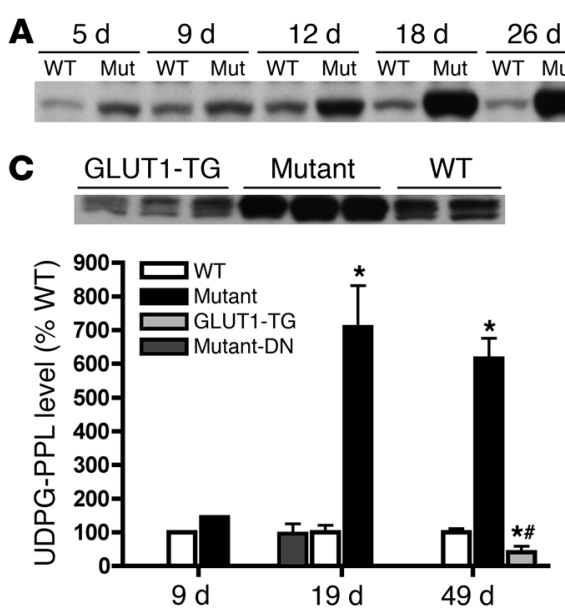

$\frac{32 d}{\text { WT Mut }} \frac{8 w k}{W T \text { Mut }}$

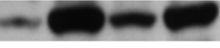

B

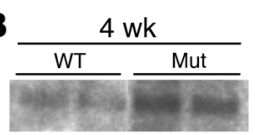

D

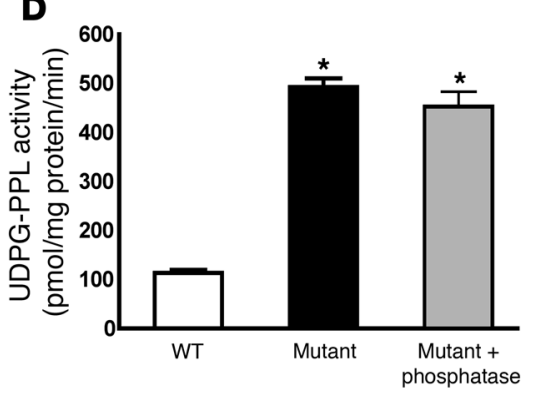

Figure 4

UDPG-PPL expression and activity in the hearts of $\gamma 2$ mutant mice. (A) Representative Western blot showing age-dependent changes in the protein amount of UDPG-PPL from 5 days to 8 weeks in hearts of $\gamma 2$ mutant (Mut) compared with age-matched WT mice. (B) Representative Northern blot showing that the mRNA level of UDPG-PPL in hearts of 4-week-old $\gamma 2$ mutant mice was 1.7 times higher than that of age-matched WT mice. (C) Representative Western blot of hearts from 7- to 10-week-old mice and protein levels of UDPG-PPL in hearts of WT mice, $\gamma 2$ mutant mice, $\gamma 2$ mutant mice crossed with mice overexpressing a dominant-negative catalytic subunit of AMPK, and transgenic mice expressing insulin-independent glucose transporter 1 (GLUT1-TG). (D) UDPG-PPL activity measured in adult hearts (7-10 weeks) before and after treatment with phosphatase. Data are mean $\pm \operatorname{SEM}(n=4-6$ per group). ${ }^{*} P<0.05$ versus age-matched WT; ${ }^{*} P<0.05$ versus age-matched mutant.

sures ANOVA). The $\gamma 2$ mutant mouse hearts showed a gradual increase in total GS activity accompanied by decreased sensitivity to G-6-P concentration. The G-6-P concentration-independent activity of GS was unaltered in at 9 and 19 days in $\gamma 2$ mutant mouse hearts, but was markedly reduced at 49 days (Figure 5A). Western blotting showed a marked band shift for GS protein (Figure 5B) that was eliminated by phosphatase treatment (data not shown), suggesting differential phosphorylation of GS in the hearts of $\gamma 2$ mutant mice compared with those of WT mice at 19 and 49 days. The progressive increase in GS phosphorylation during this period corresponded with the alterations in the relationship between GS activity and G-6-P concentration. The 2-fold increase in total GS activity in hearts of 49-day-old $\gamma 2$ mutant mice compared with agematched WT mouse hearts $(P<0.05)$ was associated with a similar increase in GS protein (Figure 5B). In the hearts of 19-day-old $\gamma 2$ mutant mice, a 2-fold increase in cardiac G-6-P level was observed compared with age-matched WT mice, which served as an effective stimulus to drive glycogen synthesis, as evidenced by the ability of the heart to maintain normal UDPG content despite significant upregulation of UDPG-PPL (Figure 5, C and D). In contrast, a higher G-6-P concentration and increased total GS protein in the hearts of 7-week-old $\gamma 2$ mutant mice failed to enhance GS activity in the physiological range of G-6-P concentration (about $0.2 \mathrm{mM}$ in normal hearts), which led to accumulation of UDPG by more than 2 -fold at this age $(P<0.05$; Figure $5, \mathrm{~A}-\mathrm{D})$. The evidence of falling glycogen synthesis at this stage was consistent with the transition from active glycogen accumulation to the plateau phase.

\section{Discussion}

In this study, we showed that altered AMPK activity in the absence of altered energy homeostasis redirected the fluxes of the cardiac meta- bolic network for fuel storage in the form of glycogen via distinct yet coordinated mechanisms (Figure 6). The resultant glycogen storage cardiomyopathy is unique in that the storage is not a result of the inability to use glycogen, but of altered metabolic regulation that, via extensive reorganization of the network, comes to a new steady state by turning over a substantially enlarged glycogen pool.

It has been well established that activation of AMPK stimulates glucose uptake, glycolysis, and fatty acid oxidation in cardiac and skeletal muscle during energetic stress such as exercise, ischemia, and hypoxia $(16-18,20)$. Although increased AMPK activity has not been observed in every mouse model overexpressing mutant $\gamma 2$-AMPK, our prior study showed that the disease phenotype can be rescued by inactivating AMPK activity (14), indicating that the functional outcome of the mutation is attributable to a gain of function of the kinase (11-14). Consistent with these findings, in the present study we observed a significantly increased rate of glucose uptake in the hearts of $\gamma 2$ mutant mice that was attenuated by ablating AMPK activity. Furthermore, the contribution of fatty acids to oxidative metabolism was higher in the hearts of $\gamma 2$ mutant mice than in those of WT mice; this is associated with increased phosphorylation of $A C C \beta$, a known target of AMPK for regulating fatty acid oxidation in muscle. While these metabolic changes were not unexpected in hearts with increased AMPK activity, their consequences in $\gamma 2$ mutant mouse hearts were distinct from those of AMPK activation during energetic stress. We showed that simultaneous increases in glucose uptake and fatty acid oxidation in the hearts of $\gamma 2$ mutant mice with normal energy status yielded a glucose-sparing effect that contributed to glycogen synthesis (Figure 6). It has previously been shown that increased glycogen synthesis occurs in the heart when enhanced oxidation of nonglucose substrate is associated with sustained glucose uptake rate under certain experimental conditions (25-27). This is because increased glucose entry and decreased glucose use for ATP production result in elevated intracellular G-6-P concentration, which serves as the source for glycogen synthesis and at the same time as a powerful allosteric stimulator of GS. Consistent with our present observations, prior studies in skeletal muscle have shown that chronic pharmacological activation of AMPK leads to glycogen storage $(28,29)$. A recent study using transgenic mice overexpressing a mutant $\gamma 1$-AMPK also showed increased AMPK activity and higher glycogen content in the muscle (30). In contrast, overexpression of the WT $\gamma 2$-AMPK in the heart that does not change AMPK activity causes a minimal disease phenotype $(11-13,15)$. These results collectively suggest that alterations of AMPK activity, rather than the isoform composition of the $\gamma$ subunit, are primarily responsible for the glycogen storage phenotype.

An unexpected finding of this study was the decrease of glycolysis from exogenous glucose in the $\gamma 2$ mutant mouse hearts, because activation of AMPK is normally associated with increased myocardial glycolysis. AMPK has previously been shown to play an important role in the phosphorylation and activation of phosphofructokinase-2 

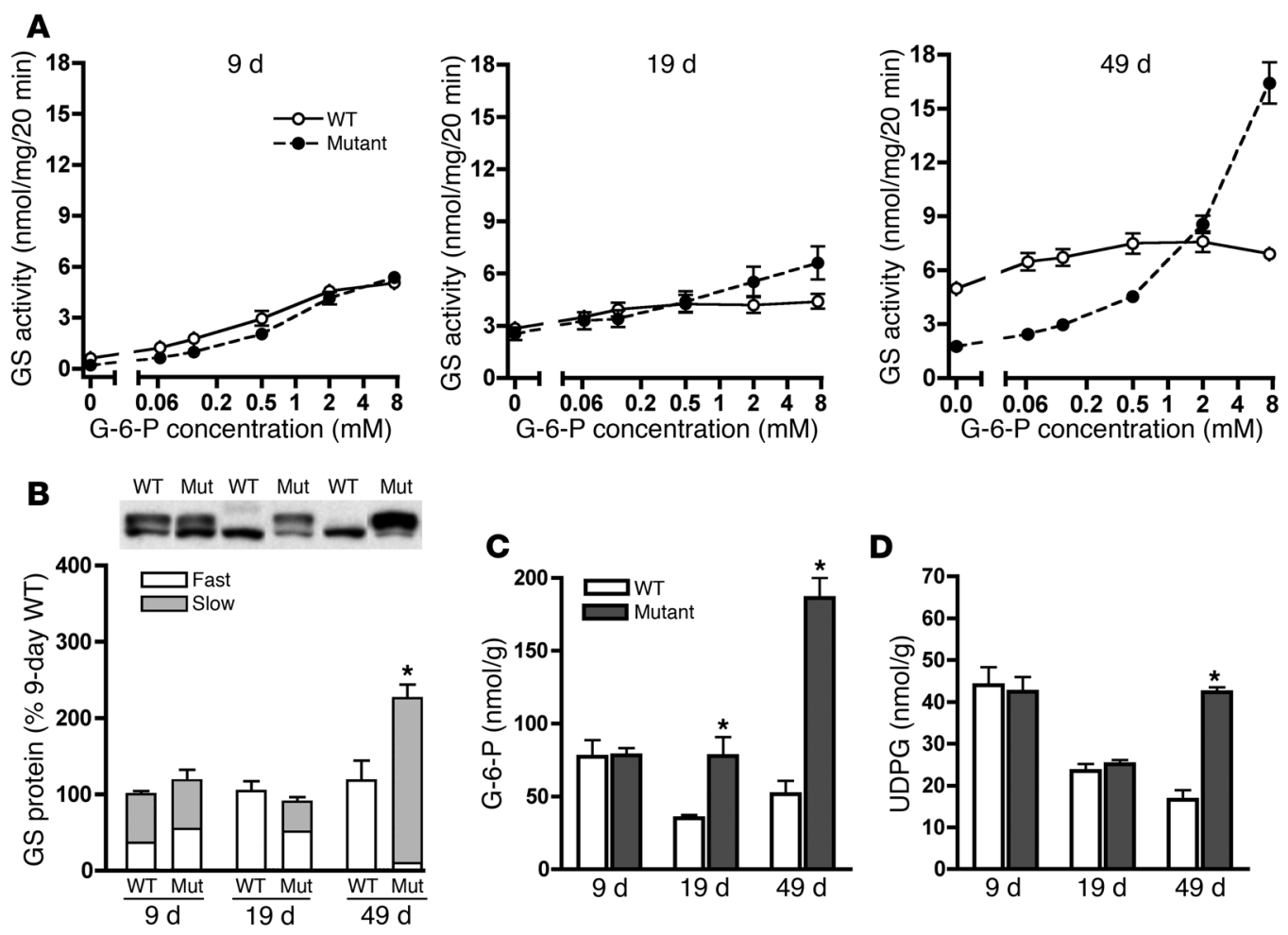

Figure 5

GS activity and its regulation in $\gamma 2$ mutant mouse hearts. (A) GS activity in cardiac tissue of WT and $\gamma 2$ mutant mice measured at G-6-P concentrations of 0.0625-7.5 mM (shown in log scale) with saturating UDPG (4.5 mM). (B) Representative Western blot of the GS protein and average quantification of the total amount of GS protein in hearts of WT and $\gamma 2$ mutant (Mut) mice at 3 time points. Bars denote relative slow- and fast-migrating fractions of the GS. Shifts of bands in gel electrophoresis and its subsequent elimination by phosphatase treatment (not shown) suggest altered phosphorylation of the GS in the hearts of $\gamma 2$ mutant mice. (C and D) Tissue content of G-6-P (C) and UDPG (D) in the hearts of $\gamma 2$ mutant mice compared with those of age-matched WT mice during the period of glycogen accumulation. Data are mean \pm SEM $(n=5-8$ per group). ${ }^{*} P<0.05$ versus age-matched WT.

(PFK-2), leading to increased synthesis of fructose-2,6-bisphosphate, a potent stimulator of the rate-limiting enzyme for the glycolytic pathway, PFK-1 $(18,31)$. However, PFK-1 activity is also regulated by other stimulators (e.g., ADP, AMP, and inorganic phosphate) and inhibitors (e.g., ATP, $\mathrm{H}^{+}$, and citrate). Under normal conditions, PFK-1 is minimally stimulated because of high concentrations of inhibitors and low concentrations of stimulators in vivo. During stress, intracellular concentrations of these regulators change coordinately, releasing the inhibition of PFK-1 and allowing glycolysis to increase in the heart (31). Aberrant AMPK activity in the hearts of $\gamma 2$ mutant mice, accompanied by no changes in other regulators of PFK-1 (14, 15 ), is likely less effective in stimulating glycolysis. Moreover, the lack of stimuli and/or demands for increasing glycolytic ATP production in the hearts of $\gamma 2$ mutant mice is combined with the active diversion of exogenous glucose toward the glycogen synthesis pathway, due to upregulation of UDPG-PPL, and thus jointly reduces the flux of exogenous glucose to glycolysis.

In addition to increased intracellular G-6-P concentration, our results suggest that increased expression and activity of UDPGPPL in the hearts of $\gamma 2$ mutant mice contribute to the "pulling" of glucose toward glycogen. In the presence of a high G-6-P concentration, an enhanced UDPG-PPL reaction served a dual role of diverting intracellular glucose toward UDPG as well as driving glycogen synthesis by increasing substrate supply via a feed- forward mechanism (Figure 6). Of note, the upregulation of UDPG-PPL (encoded by the Ugp2 gene) was an early event in the $\gamma 2$ mutant mouse heart that preceded the marked rise of myocardial glycogen content. We also showed that increased UDPG-PPL expression was independent of increased glucose uptake, because increasing glucose uptake by overexpressing glucose transporter 1 downregulated UDPG-PPL expression. Consistent with this finding, increased Ugp2 mRNA levels have also been shown in the skeletal muscle of mice (R225Q) and pigs (R200Q) bearing mutations at corresponding sites of $\gamma 3$-AMPK and presenting a glycogen storage phenotype $(32,33)$. These findings collectively suggest that upregulation of UDPG-PPL is a primary response to AMPK mutation rather than an adaptive response to altered glucose metabolism. Furthermore, our observations suggests what we believe to be a novel role of AMPK in muscle glycogen metabolism via transcriptional regulation of UDPG-PPL.

It has previously been shown that acute activation of AMPK causes phosphorylation of GS at site 2, resulting in decreased GS activity in skeletal muscles (34). However, in apparent conflict with this observation, long-term activation of AMPK increases glycogen content in skeletal muscle of normal rats and improves glycogen synthesis in insulin-resistant muscle $(35,36)$. Because our analyses of substrate metabolism were performed in the presence of insulin, they cannot address the potential alterations of insulin signaling in the hearts 


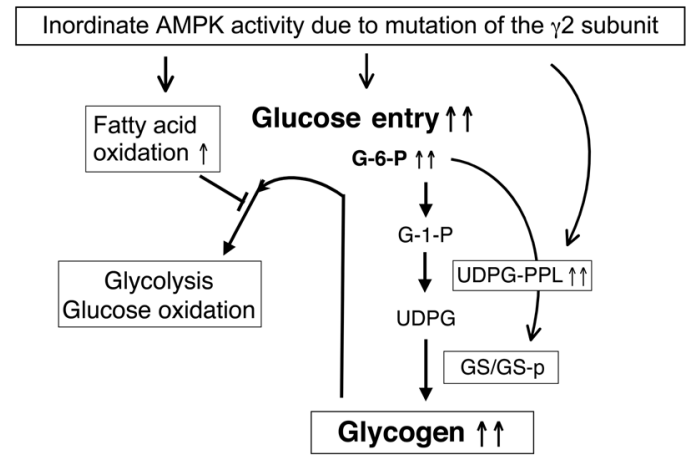

of $\gamma 2$ mutant mice. Nonetheless, we found a significant increase in the rate of insulin-independent glucose uptake in $\gamma 2$ mutant mouse hearts. It has been speculated that AMPK-mediated increases in glucose transport, after sufficiently elevating intracellular G-6-P concentrations, are able to stimulate glycogen synthesis by overcoming the inhibitory phosphorylation of GS in the skeletal muscle (28, 29, 34, 37). Our present results support such a mechanism in the heart. It is likely that increased G-6-P is particularly important for the accumulation of glycogen in the hearts of young $\gamma 2$ mutant mice when GS activity is minimally altered. Furthermore, we suggest that elevated expression of GS in the hearts of adult $\gamma 2$ mutant mice also contributes to counteract the inhibition of GS activity and, hence, sustain glycogen synthesis in the later stage.

A unique feature of the glycogen storage disease phenotype caused by mutations of $\gamma 2$-AMPK is that the heart sustains its ability to use glycogen. We have previously shown that cardiac glycogen in the hearts of $\gamma 2$ mutant mice can be used effectively in vivo to support increased energy demand during exercise and to offset decreased energy supply during substrate deprivation in isolated perfused hearts $(14,15)$. A recent study by Davies et al. found decreased branching of glycogen particles in transgenic hearts expressing a different mutant $\gamma 2$-AMPK, R531G, although the glycogen branching enzyme activity was not examined (12). We previously found normal activity of glycogen branching enzyme in N488I mutant mouse hearts (14). Furthermore, activities of the key enzymes involved in glycogenolysis, such as debranching enzyme, glycogen phosphorylase, and glycogen phosphorylase kinase, are unaltered in the N488I mutant mouse heart (14). These observations show that glycogen storage in the $\gamma 2$ mutant mouse heart is not attributable to a defective pathway for glycogenolysis as seen in majority of glycogen storage disease. It does not, however, exclude the possibility that glycogen breakdown is partially inhibited in the hearts of $\gamma 2$ mutant mice during the phase of active glycogen accumulation. Nevertheless, our results support the model that glycogen storage in the hearts of $\gamma 2$ mutant mice results from enhanced glycogen synthesis that exceeds the rate of glycogen breakdown. Furthermore, we suggest that the ultimate plateau of glycogen accumulation is set by the balance between decreased GS activity and increased contribution of glycogen to glycolytic flux. It has previously been shown that glycogen loading inhibits muscle GS activity by feedback mechanisms independent of AMPK or GS kinase $(34,38,39)$. In the present study, we found greater inhibition of GS activity in the physiological range of G-6-P concentration in the hearts of $\gamma 2$ mutant mice with peak glycogen content ( 7 weeks) compared with those during the phase of glycogen accumulation (2.5 weeks). Unlike the phase of active glycogen accumulation, a high G-6-P concentration and increased amount of GS protein in

\section{Figure 6}

Schematic summary of the remodeled substrate metabolism network leading to glycogen storage phenotype in the $\gamma 2$ mutant mouse heart. GS/GS-p, the level of GS expression and GS phosphorylation.

the hearts of 7-week-old $\gamma 2$ mutant mice failed to maintain a normal UDPG level, suggesting that the rate of glycogen synthesis becomes restricted at this stage. At the same time, glycolytic flux in the hearts of adult $\gamma 2$ mutant mice ( $90 \%$ originated from the glycogen pool) exceeded that of age-matched WT mouse hearts. Thus, the stabilization of glycogen pool size represents the dynamic balance of declined influx and active efflux of glycogen to the glycolytic pathway.

In summary, we have demonstrated that the N488I mutation of $\gamma$-AMPK modified substrate metabolism at multiple pathways via a variety of mechanisms leading to glycogen storage and eventually an active turnover of a large glycogen store. The synergistic nature of these modifications in promoting glycogen accumulation is a crucial factor in the pathogenesis of cardiac glycogen storage. Remarkably, such an extensively remodeled metabolic network is capable of maintaining energetic homeostasis in the hearts of $\gamma 2$ mutant mice and supporting increased energy demand during increases in workload (14). However, the tightly protected energy homeostasis in the $\gamma 2$ mutant mouse heart, inherent to the role of AMPK as a signaling intermediary linking energy demand to substrate metabolism, comes at the expense of developing glycogen storage cardiomyopathy. The metabolic phenotype demonstrated here not only provides the mechanisms for the pathogenesis of cardiomyopathy caused by Prkag2 mutations but also cautions against the use of nonselective AMPK activation in the absence of increased energy demand as a therapeutic strategy.

\section{Methods}

Animals. All animal experiments were approved by the Harvard Medical Area Standing Committee on Animals (Boston, Massachusetts, USA). All mice used were on an FVB background and studied at ages ranging 1.5-20 weeks as specified in each individual experiment. Transgenic mice were matched with WT littermates as controls.

Isolated heart perfusion and ${ }^{31} P$ NMR spectroscopy. Mice were heparinized and anesthetized with sodium pentobarbital (100 mg/kg i.p.), and their hearts were quickly removed. Hearts were perfused in the Langendorff mode with phosphate-free Krebs-Henseleit buffer containing $118 \mathrm{mM} \mathrm{NaCl}, 25 \mathrm{mM}$ $\mathrm{NaHCO}_{3}, 5.3 \mathrm{mM} \mathrm{KCl}, 2.5 \mathrm{mM} \mathrm{CaCl}_{2}, 1.2 \mathrm{mM} \mathrm{MgSO}_{4}, 0.5 \mathrm{mM}$ EDTA, $5 \mathrm{mM}$ glucose, and $5 \mathrm{mM}$ pyruvate at $37.5^{\circ} \mathrm{C}$ as previously described (40). All hearts were stabilized for 25 minutes at a constant perfusion pressure of $80 \mathrm{mmHg}$. A baseline ${ }^{31} \mathrm{P}$ NMR spectrum (208 scans) was collected for all hearts after stabilization. To determine the rate of glucose uptake, hearts were perfused with a buffer in which glucose was replaced with $5 \mathrm{mM}$ of the glucose analog 2-DG. Five consecutive 8 -minute ${ }^{31} \mathrm{P}$ NMR spectra were collected for determination of the time-dependent accumulation of 2-DG-phosphate (2-DG-P). The rate of glucose uptake was estimated by the slope of the fitted line as previously described $(19,41)$. During 2-DG perfusion, $1.2 \mathrm{mM} \mathrm{KH}_{2} \mathrm{PO}_{4}$ and $5 \mathrm{mM}$ pyruvate were supplied to replenish the intracellular inorganic phosphate pool and to maintain ATP synthesis.

The ${ }^{31} \mathrm{P}$ NMR resonance areas corresponding to ATP, phosphocreatine (PCr), inorganic phosphate (Pi), and 2-DG-P were fitted to Lorentzian functions and corrected for saturation (ATP, 1.0; PCr, 1.2; Pi, 1.15; 2-DG-P, 1.8). The mean value of ATP concentration previously measured by HPLC for hearts of WT or $\gamma 2$ mutant mice was used to calibrate the ATP peak area of the baseline ${ }^{31} \mathrm{P}$ NMR spectrum (14). Concentrations of other metabolites were calculated using the ratio of their peak areas to the ATP peak area 
(40). The rate of 2-DG uptake was expressed as the slope of time-dependent changes in 2-DG-P concentration.

${ }^{13} \mathrm{C} N M R$ spectroscopy and isotopomer analysis. Isolated mouse hearts were perfused with Krebs buffer containing the following substrates: $5.5 \mathrm{mM}$ glucose; $0.4 \mathrm{mM}$ mixed long chain fatty acids (bound to $1 \%$ albumin) of $60 \%$ palmitic acid, $8 \%$ palmitoleic acid, $24 \%$ oleic acid, and $8 \%$ linoleic acid; $0.38 \mathrm{mM}$ DL- $\beta$-hydroxybutyrate; $1.2 \mathrm{mM}$ lactate; and $50 \mu \mathrm{U} / \mathrm{ml}$ insulin. Myocardial oxygen consumption was determined by measuring the coronary flow rate and the oxygen pressure difference between perfusate and effluent from the pulmonary outflow tract (42). Hearts were perfused for 45 minutes with all substrates, among which 2 were ${ }^{13} \mathrm{C}$ enriched for each perfusion study as previously described (43). In one series, $\left[\mathrm{U}-{ }^{13} \mathrm{C}\right]$ fatty acid and $\left[3-{ }^{13} \mathrm{C}\right]$ lactate were used to determine the relative contributions of fatty acid and lactate to acetyl-CoA. Identical experiments with enriched $\left[\mathrm{U}-{ }^{13} \mathrm{C}\right]$ glucose and $\left[2,4-{ }^{13} \mathrm{C}\right] \beta$-hydroxybutyrate determined the relative contributions of these 2 substrates. Protondecoupled ${ }^{13} \mathrm{C}$ NMR $(9.4 \mathrm{~T}, 102.8 \mathrm{MHz})$ spectra of cardiac tissue extracts were acquired using a 3-mm NMR probe (Varian Medical Systems). The contributions of each labeled substrate and the total of unlabeled substrates to the oxidative metabolism were determined using the ${ }^{13} \mathrm{C}$ isotopomers peak areas of the $\mathrm{C} 3$ and $\mathrm{C} 4$ of glutamate by modeling the tricarboxylic acid cycle fluxes as previously described $(44,45)$. The contribution from unlabeled endogenous substrates was determined as the difference from $100 \%$.

In a separate cohort of hearts, dynamic incorporation of $\left[1-{ }^{13} \mathrm{C}\right]$ glucose into the $\mathrm{C}-1$ resonance of glycogen was assessed. ${ }^{13} \mathrm{C}$ NMR spectra of the perfused adult hearts ( $7-10$ weeks old) were averaged for 8-minute intervals until the ${ }^{13} \mathrm{C}$ glycogen peak area reached a steady state (approximately 2 hours). The spectra were calibrated using an external standard of $\left[1-{ }^{13} \mathrm{C}\right]$ glucose. Due to their much smaller heart size, the spectra of 3-weekold mouse hearts were averaged for 24-minute intervals during the 2-hour perfusion. Hearts were freeze-clamped at the end of perfusion for biochemical assays and ${ }^{13} \mathrm{C}$ isotopomer analysis as described above.

Biochemical assays and Northern and Western blotting. Ventricular tissue (approximately $10 \mathrm{mg}$ ) was homogenized for 10 seconds at $4^{\circ} \mathrm{C}$ in potassium phosphate buffer containing $1 \mathrm{mmol} / \mathrm{l}$ EDTA and $1 \mathrm{mmol} / 1 \beta$-mercaptoethanol, pH 7.4 (final concentration, $5 \mathrm{mg}$ tissue/ml). Aliquots were removed for assays of protein content using BSA as the standard (46). Tissue activities of UDPG-PPL were determined before and after treatment with phosphatase as previously described (47). GS activity was determined as previously described with increasing concentrations of G-6-P $(0,0.0625$, $0.125,0.5,2.0$, and $7.5 \mathrm{mM}$ ) in the presence of $4.5 \mathrm{mM}$ UDPG (48).
Myocardial glycogen content was determined by measuring the amount of glucose released from glycogen with a glucose assay kit (catalog no. GAHK-20; Sigma-Aldrich). An alkaline extraction procedure was used to separate glycogen and exogenous glucose in the tissue (49). Cardiac content of G-6-P and UDPG was determined in freeze-clamped tissue by the methods of Lowry and Passonneau (50). All reagents were purchased from Sigma-Aldrich and were at least analytical grade.

The level of mRNA for UDPG-PPL in the heart was determined by Northern blotting. Cardiac tissue lysates were used for immunoblotting with antibodies against ACC (Cell Signaling Technology) and phosphorylated ACC (Upstate USA Inc.), GS (Chemicon International), and UDPG-PPL (kindly provided by J.C. Lawrence, University of Virginia School of Medicine, Charlottseville, Virginia, USA).

Statistics. Data are presented as mean \pm SEM. One-way ANOVA was performed for multiple-group comparisons, and unpaired Student's $t$ test was used for comparisons between 2 groups. Two-way repeated-measures ANOVA was used to compare the time-dependent changes and the doseresponse relationships obtained during ${ }^{13} \mathrm{C}$-glycogen labeling experiments and GS activity assays, respectively. For the dose-response relationship between G-6-P concentration and GS activity, 2-way repeated-measures ANOVA was used to determine whether the GS activity is affected by G-6-P concentration, genotype, or interaction of concentration and genotype (i.e., does concentration have the same effect on both genotypes?). For all comparisons, a value of $P<0.05$ was considered to be significant.

\section{Acknowledgments}

This work is supported by NIH grants AR45670 and DK68626 (to L.J. Goodyear), HL52320 (to J.S. Ingwall), HL46033 (to J.A. Balschi), and HL67970 and HL59246 (to R. Tian), as well as by grants from the National Heart, Lung, and Blood Institute, NIH (to J.G. Seidman). R. Tian is an Established Investigator of the American Heart Association. I. Luptak was a recipient of the American Heart Association postdoctoral fellowship.

Received for publication October 16, 2006, and accepted in revised form February 13, 2007.

Address correspondence to: Rong Tian, NMR Laboratory, Division of Cardiovascular Medicine, Brigham and Women's Hospital, 221 Longwood Avenue, Room 252, Boston, Massachusetts 02115, USA. Phone: (617) 732-6994; Fax: (617) 732-6990; E-mail: rtian@ rics.bwh.harvard.edu.
1. Hardie, D.G., and Carling, D. 1997. The AMP-activated protein kinase--fuel gauge of the mammalian cell? Eur. J. Biochem. 246:259-273.

2. Corton, J.M., Gillespie, J.G., and Hardie, D.G. 1994. Role of the AMP-activated protein kinase in the cellular stress response. Curr. Biol. 4:315-324.

3. Kemp, B.E., et al. 1999. Dealing with energy demand: the AMP-activated protein kinase. Trends Biochem. Sci. 24:22-25.

4. Arad, M., et al. 2002. Constitutively active AMP kinase mutations cause glycogen storage disease mimicking hypertrophic cardiomyopathy. J. Clin. Invest. 109:357-362. doi:10.1172/JCI200214571.

5. Blair, E., et al. 2001. Mutations in the gamma(2) subunit of AMP-activated protein kinase cause familial hypertrophic cardiomyopathy: evidence for the central role of energy compromise in disease pathogenesis. Hum. Mol. Genet. 10:1215-1220.

6. Gollob, M.H., et al. 2001. Identification of a gene responsible for familial Wolff-Parkinson-White syndrome. N. Engl. J. Med. 344:1823-1831.

7. Arad, M., et al. 2005. Glycogen storage diseases pre- senting as hypertrophic cardiomyopathy. N. Engl.J. Med. 352:362-372.

8. Milan, D., et al. 2000. A mutation in PRKAG3 associated with excess glycogen content in pig skeletal muscle. Science. 288:1248-1251.

9. Barnes, B.R., et al. 2004. The 5'-AMP-activated protein kinase gamma3 isoform has a key role in carbohydrate and lipid metabolism in glycolytic skeletal muscle. J. Biol. Chem. 279:38441-38447.

10. Yu, H., et al. 2006. Muscle-specific overexpression of wild type and R225Q mutant AMP-activated protein kinase gamma3-subunit differentially regulates glycogen accumulation. Am. J. Physiol. Endocrinol. Metab. 291:E557-E565.

11. Arad, M., et al. 2003. Transgenic mice overexpressing mutant PRKAG2 define the cause of WolffParkinson-White syndrome in glycogen storage cardiomyopathy. Circulation. 107:2850-2856.

12. Davies, J.K., et al. 2006. Characterization of the role of gamma2 R531G mutation in AMP-activated protein kinase in cardiac hypertrophy and WolffParkinson-White syndrome. Am. J. Physiol. Heart
Circ. Physiol. 290:H1942-H1951.

13. Sidhu, J.S., et al. 2005. Transgenic mouse model of ventricular preexcitation and atrioventricular reentrant tachycardia induced by an AMP-activated protein kinase loss-of-function mutation responsible for Wolff-Parkinson-White syndrome. Circulation. 111:21-29.

14. Ahmad, F., et al. 2005. Increased alpha2 subunitassociated AMPK activity and PRKAG2 cardiomyopathy. Circulation. 112:3140-3148.

15. Zou, L., et al. 2005. N488I mutation of the gamma2-subunit results in bidirectional changes in AMP-activated protein kinase activity. Circ. Res. 97:323-328.

16. Hayashi, T., et al. 2000. Metabolic stress and altered glucose transport: activation of AMP-activated protein kinase as a unifying coupling mechanism. Diabetes. 49:527-531.

17. Kudo, N., Barr, A.J., Barr, R.L., Desai, S., and Lopaschuk, G.D. 1995. High rates of fatty acid oxidation during reperfusion of ischemic hearts are associated with a decrease in malonyl-CoA lev- 
els due to an increase in $5^{\prime}$-AMP-activated protein kinase inhibition of acetyl-CoA carboxylase. J. Biol. Chem. 270:17513-17520.

18. Marsin, A.S., et al. 2000. Phosphorylation and activation of heart PFK-2 by AMPK has a role in the stimulation of glycolysis during ischaemia. Curr. Biol. 10:1247-1255.

19. Tian, R., Musi, N., D’Agostino, J., Hirshman, M.F., and Goodyear, L.J. 2001. Increased adenosine monophosphate-activated protein kinase activity in rat hearts with pressure-overload hypertrophy. Circulation. 104:1664-1669.

20. Russell, R.R., 3rd, et al. 2004. AMP-activated protein kinase mediates ischemic glucose uptake and prevents postischemic cardiac dysfunction, apoptosis, and injury. J. Clin. Invest. 114:495-503. doi:10.1172/JCI200419297.

21. Lyons, G.E., Schiaffino, S., Sassoon, D., Barton, P., and Buckingham, M. 1990. Developmental regulation of myosin gene expression in mouse cardiac muscle. J. Cell Biol. 111:2427-2436.

22. Subramaniam, A., et al. 1991. Tissue-specific regulation of the alpha-myosin heavy chain gene promoter in transgenic mice. J. Biol. Chem. 266:24613-24620.

23. Gulick, J., Subramaniam, A., Neumann, J., and Robbins, J. 1991. Isolation and characterization of the mouse cardiac myosin heavy chain genes. J. Biol. Chem. 266:9180-9185.

24. Liao, R., et al. 2002. Cardiac-specific overexpression of GLUT1 prevents the development of heart failure attributable to pressure overload in mice. Circulation. 106:2125-2131.

25. Depre, C., Rider, M.H., and Hue, L. 1998. Mechanisms of control of heart glycolysis. Eur. J. Biochem. 258:277-290.

26. Williamson, J.R. 1965. Glycolytic control mechanisms. I. Inhibition of glycolysis by acetate and pyruvate in the isolated, perfused rat heart. J. Biol. Chem. 240:2308-2321.

27. Laughlin, M.R., Taylor, J., Chesnick, A.S., and Balaban, R.S. 1994. Nonglucose substrates increase glycogen synthesis in vivo in dog heart. Am. J. Physiol. 267:H219-H223.

28. Buhl, E.S., et al. 2001. Chronic treatment with 5-aminoimidazole-4-carboxamide-1-beta-D-ribofurano- side increases insulin-stimulated glucose uptake and GLUT4 translocation in rat skeletal muscles in a fiber type-specific manner. Diabetes. 50:12-17.

29. Winder, W.W., et al. 2000. Activation of AMP-activated protein kinase increases mitochondrial enzymes in skeletal muscle. J. Appl. Physiol. 88:2219-2226.

30. Barre, L., et al. 2007. A genetic model for the chronic activation of skeletal muscle AMP-activated protein kinase leads to glycogen accumulation. Am. J. Physiol. Endocrinol. Metab. 292:E802-E811.

31. Nascimben, L., et al. 2004. Mechanisms for increased glycolysis in the hypertrophied rat heart. Hypertension. 44:662-667.

32. Hedegaard, J., et al. 2004. UDP-glucose pyrophosphorylase is upregulated in carriers of the porcine $\mathrm{RN}$ - mutation in the AMP-activated protein kinase. Proteomics. 4:2448-2454.

33. Nilsson, E.C., et al. 2006. Opposite transcriptional regulation in skeletal muscle of AMP-activated protein kinase gamma3 R225Q transgenic versus knock-out mice. J. Biol. Chem. 281:7244-7252.

34. Jorgensen, S.B., et al. 2004. The alpha2-5'AMP-activated protein kinase is a site 2 glycogen synthase kinase in skeletal muscle and is responsive to glucose loading. Diabetes. 53:3074-3081.

35. Holmes, B.F., Kurth-Kraczek, E.J., and Winder, W.W. 1999. Chronic activation of 5'-AMP-activated protein kinase increases GLUT-4, hexokinase, and glycogen in muscle. J. Appl. Physiol. 87:1990-1995.

36. Olsen, G.S., and Hansen, B.F. 2002. AMP kinase activation ameliorates insulin resistance induced by free fatty acids in rat skeletal muscle. Am. J. Physiol. Endocrinol. Metab. 283:E965-E970.

37. Skurat, A.V., Dietrich, A.D., and Roach, P.J. 2000. Glycogen synthase sensitivity to insulin and glucose-6-phosphate is mediated by both NH2- and $\mathrm{COOH}$-terminal phosphorylation sites. Diabetes. 49:1096-1100.

38. Nielsen, J.N., et al. 2001. Glycogen synthase localization and activity in rat skeletal muscle is strongly dependent on glycogen content. J. Physiol. 531:757-769.

39. Wojtaszewski, J.F., et al. 2003. Regulation of 5'AMPactivated protein kinase activity and substrate utilization in exercising human skeletal muscle. Am.J.
Physiol. Endocrinol. Metab. 284:E813-E822.

40. Xing, Y., et al. 2003. Glucose metabolism and energy homeostasis in mouse hearts overexpressing dominant negative alpha 2 subunit of AMP-activated protein kinase. J. Biol. Chem. 278:28372-28377.

41. Abel, E.D., et al. 1999. Cardiac hypertrophy with preserved contractile function after selective deletion of GLUT4 from the heart. J. Clin. Invest. 104:1703-1714.

42. Saupe, K.W., Spindler, M., Tian, R., and Ingwall, J.S. 1998. Impaired cardiac energetics in mice lacking muscle-specific isoenzymes of creatine kinase. Circ. Res. 82:898-907.

43. Luptak, I., et al. 2005. Decreased contractile and metabolic reserve in peroxisome proliferator-activated receptor-alpha-null hearts can be rescued by increasing glucose transport and utilization. Circulation. 112:2339-2346.

44. Malloy, C.R., Sherry, A.D., and Jeffrey, F.M. 1988. Evaluation of carbon flux and substrate selection through alternate pathways involving the citric acid cycle of the heart by $13 \mathrm{C}$ NMR spectroscopy. J. Biol. Chem. 263:6964-6971.

45. Malloy, C.R., Sherry, A.D., and Jeffrey, F.M. 1990. Analysis of tricarboxylic acid cycle of the heart using 13C isotope isomers. Am. J. Physiol. 259:H987-H995.

46. Lowry, O.H., Rosebrough, M.J., Farr, A.L., and Randall, R.J. 1951. Protein measurement with the foline reagent. J. Biol. Chem. 193:265-275.

47. Roach, P.J., Warren, K.R., and Atkinson, D.E. 1975. Uridine diphosphate glucose synthase from calf liver: determinants of enzyme activity in vitro. Biochemistry. 14:5445-5450.

48. Thomas, J.A., Schlender, K.K., and Larner, J. 1968. A rapid filter paper assay for UDPglucose-glycogen glucosyltransferase, including an improved biosynthesis of UDP-14C-glucose. Anal. Biochem. 25:486-499.

49. Passonneau, J.V., and Lauderdale, V.R. 1974. A comparison of three methods of glycogen measurement in tissues. Anal. Biochem. 60:405-412.

50. Lowry, O.H., and Passonneau, J.V. 1972. A collection of metabolite assays. Academic Press. New York, New York, USA. 146-218. 